\title{
Toy superstrings
}

\author{
Marco Corvi and V. Alan Kostelecký \\ Physics Department, Indiana University, Bloomington, Indiana 47405 \\ Peter Moxhay \\ Physics Department, University of Colorado, Boulder, Colorado 80309
}

(Received 31 August 1988)

\begin{abstract}
Superstrings with critical dimension two and two real bosonic spacetime coordinates may serve as useful toy models for the study of string properties. We present details of several such toy superstrings including open, closed, and heterotic models. Conformal methods are used to establish the spectrum and dimensionality. The spin fields are provided and Becchi-Rouet-Stora-Tyutin- (BRST-) invariant vertex operators are constructed. Four-point tree-level and four-point one-loop amplitudes in these models are obtained. The closed and heterotic toy superstrings are shown to be modular invariant to this order.
\end{abstract}

\section{INTRODUCTION}

Superstring theory ${ }^{1,2}$ is a rich and deep mathematical structure with important potential applications to theoretical physics. It provides an approach that may be consistent at the quantum level to the unification of all fundamental forces and particles.

As prime candidates for a unified theory of nature incorporating quantum gravity, superstring theories are evidently highly complex. Part of the complexity stems from the infinite number of particle states involved. The large number of dimensions that occurs further complicates the study of string physics. The critical dimension for superstring theories is ten, while that for bosonic strings is 26. Most mathematical tools available are inadequate for a nonperturbative investigation.

The development of toy superstring models that have at least some of the aspects of ten-dimensional superstring theories may yield insights into the physics of the latter. One goal of this paper is to establish several such models.

The key idea we have used in the construction of toy superstrings is to restrict ourselves to two real spacetime dimensions. Calculations in two dimensions are typically much easier than in higher dimensions. An added benefit is that the spectra of such toy superstrings in some cases contain a finite number of particles. We are anticipating the possibility of addressing questions with the toy models that are of great interest but that in higherdimensional superstrings would involve prohibitive calculations. The answers to such questions might prove clues to the behavior and nature of ten-dimensional superstrings.

An open superstring theory with critical dimension two has previously been presented in the literature. ${ }^{3-5}$ The model has $N=2$ supersymmetry. There are two sets of spacetime bosons: $X^{\mu}$ and $Y^{\mu}$. The presence of four bosonic coordinates in a theory with $\mathrm{SO}(1,1)$ as Lorentz group means that the interpretation of the model is subtle. For example, the coordinates are naturally treated as complex, as are the momenta. An attempt has been made to reinterpret this model as a four-dimensional theory in Ref. 6. It has also been used to explain the origin of the world sheet in higher-dimensional superstrings. ${ }^{7}$

In addition to the open superstring, further twodimensional models are possible. A closed superstring is naturally constructed from the action of the open one by imposing the standard closed-string boundary conditions. Closed-superstring models with generalized boundary conditions have been considered in Refs. 8 and 9. The existence of other simple theories is suggested by requirements of conformal anomaly cancellation. ${ }^{10}$ These models include heterotic strings with $(2,0)$ or $(2,1)$ world-sheet supersymmetry; in fact, the possibility of heterotic strings with critical dimension two was noted in Ref. 11. Properties of some models with $(2,0)$ world-sheet supersymmetry have been explored in Ref. 7. Further possibilities include compactified models in higher critical dimensions.

Motivated by the desire for toy superstring theories without interpretational difficulties, we seek models with critical dimension two that have only two real bosonic coordinates playing the role of spacetime dimensions. Models of this type may be constructed from some of the ones mentioned above by requiring compactification of the $Y$ directions. We take the radius of compactification to zero wherever the resulting projection is compatible with modular invariance. Furthermore, we restrict our closed-string models to the sectors periodic in the bosonic coordinates $X$ and $Y$. These procedures allow an interpretation of two-dimensional superstring models that is both tractable and suited to our purposes. The resulting models are somewhat different from the $N=2$ superstrings with critical dimension two that have previously been discussed in the literature.

The compactification projection may be imposed by the following prescription. Take the $Y$ coordinates to be valued on a torus of radius $\epsilon$, defined as the quotient of $\mathbb{R}^{2}$ by a lattice $\Lambda_{0}$. The corresponding $Y$ momenta are thus quantized in multiples of $1 / \epsilon$. In the limit as $\epsilon$ tends to zero, we allow only states with finite energies to play 
the role of in or out states in the quantum scattering problem. In effect, this compactification restricts the $Y$ momenta of all states to be zero and thus imposes translational invariance in the $Y$ directions. Note, however, that caution is needed in the computation of loop amplitudes because there can be residual effects from the states with infinite energies. Note also that for our heterotic models the limit of zero $Y$ momenta cannot be imposed: modular invariance requires a finite and fixed radius of compactification. These points are discussed in more detail in Sec. IV.

In this paper, we formulate several toy superstrings and study their basic properties. The models discussed include oriented and unoriented open superstrings both with and without Paton-Chan factors, ${ }^{12}$ closed superstrings, and $(2,0)$ heterotic strings. In Sec. II these models are presented. The analysis of the spectrum and dimensionality for each toy model is performed using conformal techniques. The vertex operators discussed include ones for the scalar and spin-field vertices. Section III contains the derivation of tree-level scattering amplitudes for the models. In Sec. IV four-point one-loop amplitudes are studied. The closed and heterotic toy superstring amplitudes are shown to be modular invariant to this order. Section V concludes.

We remark that wherever possible we have followed the notation of Ref. 2. In particular, the two-dimensional metric is $\eta_{00}=-1, \eta_{11}=1$ and the two-dimensional gamma matrices $\rho^{\mu}$ are $\rho^{0}=\sigma^{2}, \rho^{1}=i \sigma^{1}$. Note also that various authors use different definitions of Neveu-Schwarz (NS) and Ramond (R) sectors; we refer to the scalar-field sector as NS and the spin-field one as $\mathbf{R}$.

\section{TWO-DIMENSIONAL SUPERSTRING MODELS}

\section{A. Open superstring}

The basic open toy superstring ${ }^{3-5}$ consists of two $D$ dimensional bosonic coordinates $X^{\mu}$ and $Y^{\mu}$ and two two-component fermionic ones $\psi^{j \mu}, j=1,2$. The gaugefixed action is

$S=\frac{-1}{4 \pi \alpha^{\prime}} \int d^{2} \sigma\left(\partial_{\alpha} X \cdot \partial^{\alpha} X+\partial_{\alpha} Y \cdot \partial^{\alpha} Y-i \bar{\psi}^{j} \cdot \rho^{\alpha} \partial_{\alpha} \psi^{j}\right)$.

This action is invariant under conformal transformations, supersymmetry transformations and $O(2)$ rotations. The conformal symmetry is the remnant after gauge fixing of the invariance under reparametrizations of the world sheet, common to every string theory. The supersymmetry relates the bosonic and the fermionic components:

$$
\begin{aligned}
& \delta X^{\mu}=\bar{\epsilon}^{j} \psi^{j \mu}, \quad \delta Y^{\mu}=\varepsilon^{j k} \bar{\epsilon}^{j} \psi^{k \mu}, \\
& \delta \psi^{j \mu}=-i \rho^{\alpha} \partial_{\alpha} X^{\mu} \epsilon^{j}+i \varepsilon^{j k} \rho^{\alpha} \partial_{\alpha} Y^{\mu} \epsilon^{k},
\end{aligned}
$$

where $\varepsilon^{j k}$ is antisymmetric with $\varepsilon^{12}=1$ and $\epsilon^{j}$ are Grassmann-valued constants. The $\mathbf{O}(2)$ symmetry involves rotations among the two fermionic coordinates:

$$
\psi^{j}=R^{j k} \psi^{k}, \quad R \in \mathrm{O}(2) .
$$

The choice of boundary conditions specifies the string model. A consistent set of boundary conditions for the open string is

$$
\begin{aligned}
& \partial_{1} X=\partial_{1} Y=0 \text { at } \sigma^{1}=0, \pi, \\
& \psi_{+}^{j}=\psi_{-}^{j} \quad \text { at } \sigma^{1}=0, \quad \psi_{+}^{j}=R_{\phi}^{j k} \psi_{-}^{k} \text { at } \sigma^{1}=\pi,
\end{aligned}
$$

where the components of $\psi^{j}$ are denoted as $\psi_{-}^{j}$ and $\psi_{+}^{j}$ and where $R_{\phi} \in \mathrm{O}(2)$ rotates the coordinates by an angle $\phi$. The determinant of $R_{\phi}$ distinguishes between the Ramond (R) and the Neveu-Schwarz (NS) sectors, being - 1 for the former and +1 for the latter. Within each sector different choices of $\phi$ yield equivalent descriptions of the theory, ${ }^{3}$ related by an $\mathrm{SO}(2)$ rotation.

For the NS sector we select $\phi=\pi$. Then, setting $\alpha^{\prime}=\frac{1}{2}$, the normal-mode expansions of the string coordinates have the usual form

$$
\begin{aligned}
& X(\sigma)=q^{X}+p^{X} \sigma^{0}+i \sum_{n \neq 0} \frac{\alpha_{n}^{X}}{n} \cos \left(n \sigma^{1}\right) e^{-i n \sigma^{0}} \\
& Y(\sigma)=q^{Y}+p^{Y} \sigma^{0}+i \sum_{n \neq 0} \frac{\alpha_{n}^{Y}}{n} \cos \left(n \sigma^{1}\right) e^{-i n \sigma^{0}} \\
& \psi_{ \pm}^{j}(\sigma)=\frac{1}{\sqrt{2}} \sum_{r \in \mathbb{Z}+1 / 2} b_{r}^{j} e^{-i r\left(\sigma^{0} \pm \sigma^{1}\right)} .
\end{aligned}
$$

Canonical quantization of the string coordinates $X, Y$, and $\psi^{j}$ results in the nonvanishing commutation relations

$$
\begin{aligned}
& {\left[\alpha_{m}^{X \mu}, \alpha_{n}^{X v}\right]=\left[\alpha_{m}^{Y \mu}, \alpha_{n}^{Y v}\right]=m \delta_{m+n} \eta^{\mu v}} \\
& {\left[q^{X \mu}, p^{X v}\right]=\left[q^{Y \mu}, p^{Y v}\right]=i \eta^{\mu v}} \\
& \left\{b_{r}^{j \mu}, b_{s}^{k v}\right\}=\delta_{r+s} \eta^{\mu v}
\end{aligned}
$$

The analysis of the dimensionality and spectrum of the theory can be carried out using conformal techniques. ${ }^{13-23}$ The action becomes

$S=\frac{1}{4 \pi \alpha^{\prime}} \int d z d \bar{z}\left(\partial X \cdot \bar{\partial} X+\partial Y \cdot \bar{\partial} Y+\hat{\psi}^{j} \cdot \bar{\partial} \hat{\psi}^{j}\right)$

Here, we have rotated to Euclidean time, $\sigma^{2}=i \sigma^{0}$, and used the conformal variables $z=e^{\sigma^{2}+i \sigma^{1}}, \bar{z}=e^{\sigma^{2}-i \sigma^{1}}$. We have also introduced the conformal fermionic fields $\hat{\psi}^{j}(z)=\sqrt{2 / z} \psi^{j}(-i \ln z)$, where $\psi^{j}(\sigma)$ is defined as $\psi_{+}^{j}\left(\sigma^{0},+\sigma^{1}\right)$ for $\sigma^{1}>0$ and as $\psi_{-}^{j}\left(\sigma^{0},-\sigma^{1}\right)$ for $\sigma^{1}<0$.

The Noether currents associated with the symmetries of the action are

$$
\begin{aligned}
& L=-\frac{1}{2}\left(\partial X \cdot \partial X+\partial Y \cdot \partial Y+\hat{\psi}^{j} \cdot \partial \hat{\psi}^{j}\right), \\
& G^{j}=i \hat{\psi}^{j} \cdot \partial X+i \varepsilon^{j k} \hat{\psi}^{k} \cdot \partial Y, \quad T=-i \hat{\psi}^{1} \cdot \hat{\psi}^{2},
\end{aligned}
$$

where $L, G^{j}$, and $T$ are the currents of the conformal symmetry, the supersymmetry, and the $\mathrm{O}(2)$ symmetry, respectively. These satisfy an $N=2$ superconformal algebra $^{3,4}$ 


$$
\begin{aligned}
& T(z) T(w) \sim \frac{D}{(z-w)^{2}}, \\
& G^{j}(z) T(w) \sim \frac{-i}{z-w} \varepsilon^{j k} G^{k}(\omega), \\
& G^{j}(z) G^{k}(w) \sim 2 \delta^{j k}\left[\frac{D}{(z-w)^{3}}+\frac{1}{z-w} L(w)\right] \\
& \quad+i \varepsilon^{j k}\left[\frac{2}{(z-w)^{2}} T(w)+\frac{1}{z-w} \partial T(w)\right], \\
& L(z) T(w) \sim \frac{1}{(z-w)^{2}} T(w)+\frac{1}{z-w} \partial T(w), \\
& L(z) G(w) \sim \frac{3}{2} \frac{1}{(z-w)^{2}} G^{j}(w)+\frac{1}{z-w} \partial G^{j}(w), \\
& L(z) L(w) \sim \frac{3}{2} \frac{D}{(z-w)^{4}}+\frac{2}{(z-w)^{2}} L(w)+\frac{1}{z-w} \partial L(w) .
\end{aligned}
$$

The string coordinates are supplemented by ghost fields $c$ and $b$ for the conformal symmetry, $\gamma^{j}$ and $\beta^{j}$ for the two supersymmetries, and $C$ and $B$ for the $O(2)$ symmetry. Their bosonized expressions are

$$
\begin{aligned}
& c=e^{\sigma}, \quad b=e^{-\sigma}, \\
& \gamma^{j}=e^{\phi^{j}} \eta^{j}, \quad \beta^{j}=\partial \xi^{j} e^{-\phi^{j}}, \\
& C=e^{\Sigma}, \quad B=e^{-\Sigma} .
\end{aligned}
$$

From Eq. (2.9), we see that the conformal weights of $L$, $G^{j}$, and $T$ are $2, \frac{3}{2}$, and 1 , respectively. Consequently, the central charges for the ghosts $c, \gamma^{j}$, and $C$ are $-26,11$, and -2 , respectively. The conformal anomaly for the ghost system is $c=-6$. This cancels the conformal anomaly of the string coordinates provided that the dimensionality is $D=2$.

As usual, the cancellation of the conformal anomaly can be viewed as arising from the nilpotency of a BecchiRouet-Stora-Tyutin (BRST) operator $Q$ associated with the $N=2$ superconformal algebra (2.9) and the ghost system (2.10). This operator is presented in Refs. 24 and 25, where its nilpotency is explicitly verified.

As discussed in the Introduction, we are interested in the toy-superstring limit of this model for which there is translational invariance in the $Y$ directions. We thus assume that the $Y$ coordinates take values in a compactified space on a torus of arbitrarily small radius $\epsilon$, given as the quotient of $\mathbb{R}^{2}$ by a lattice $\Lambda_{0}$. The $Y$ momentum of the states is quantized in multiples of $1 / \epsilon$, so in the limit of vanishing $\epsilon$ only states with $p^{Y}=0$ remain at finite energies. The spectrum of the model is restricted to these states.

Since the open toy superstring has two bosonic spacetime coordinates and critical dimension two, it admits of a simple physical interpretation. The bosonic part of the string may be viewed as a spatially one-dimensional object lying in one spatial dimension, i.e., a map of $\mathbb{R}^{1}$ to $\mathbb{R}^{1}$. As the longitudinal oscillations are decoupled and as no transversal oscillations are possible, on physical grounds one expects the only relevant parameter to be the location of the center of mass. Therefore, once the projection to the toy model has been applied, only one bosonic degree of freedom should remain. The world-sheet supersymmetry provides a partner state. We next show that this expectation is indeed satisfied.

The physical states are built up over the sl(2)-invariant vacuum state $|0\rangle$. The lowest NS state, $|0\rangle_{\mathrm{NS}}$, is obtained applying the ghosts $e^{-\phi^{1}}$ and $e^{-\phi^{2}}$ to $|0\rangle$. The corresponding vertex operator is

$$
V^{(\mathrm{NS})}(k)=e^{-\phi^{1}-\phi^{2}} e^{i k \cdot X},
$$

where $k$ is the $X$-momentum of the state. The condition $k^{2}=0$ ensures that the vertex $V^{(N S)}(k)$ has the correct conformal dimension, $h=1$. When multiplied by $c$ or integrated over $z$, this vertex is BRST invariant.

Higher states are obtained by applying a suitable number of fermionic fields $\psi^{j}$. To give a physical state, these operators must appear in the combination $\zeta_{\mu \nu} \psi^{j \mu} \psi^{k v}$, where $\zeta_{\mu \nu}$ is a polarization tensor satisfying $\zeta_{\mu v} k^{\mu}=\zeta_{\mu v} k^{v}=0$. In two dimensions these requirements are very stringent: $j$ and $k$ must be different. The resulting allowed physical states have zero norm. Therefore, any higher state in the NS sector is spurious. We conclude that there is only one physically relevant NS state, the vacuum $|0\rangle_{\mathrm{NS}}$.

To describe the content of the $R$ sector we construct the spin fields. ${ }^{26-32,19-23}$ First, bosonize the fermionic coordinates as follows:

$$
\widehat{\psi}^{j \pm} \equiv \frac{1}{\sqrt{2}}\left(\hat{\psi}^{j 1} \pm \hat{\psi}^{j 0}\right)=e^{ \pm \varphi^{j}} c\left( \pm \hat{e}^{j}\right)
$$

where $c\left( \pm \hat{e}^{j}\right)$ is a cocycle operator, ensuring correct statistics. Then, the spin fields are

$$
S^{A, a}=e^{A \cdot \varphi+a \cdot \phi} c(A, a),
$$

where $A \equiv\left(A^{1}, A^{2}\right)=\left( \pm \frac{1}{2}, \pm \frac{1}{2}\right)$ is the coordinate part of the vector associated with the spin field, and $a=\left( \pm \frac{1}{2}, \pm \frac{1}{2}\right)$ is the ghost part.

The spin fields split into two classes of opposite chiralities, according to the parity of the number of minus signs in $(A, a)$. The operator-product expansions (OPE's) between operators from different classes have fractional powers of $(z-w)$. We must therefore select one class. In each class there are two spin fields with conformal dimension appropriate for creation of a state when applied to the sl(2)-invariant vacuum $|0\rangle$. These have $a=\left(-\frac{1}{2},-\frac{1}{2}\right)$. For simplicity, we write $a=(-,-)$.

The integrand of the BRST-invariant vertex operator that creates a physical state is

$$
V_{(-,-)}^{(R)}(k, u)=\sum_{A} u_{A} S^{(A,-,-)} e^{i k \cdot X}
$$

where $u_{A}$ describes the polarization of the state. For a physical state, $u_{A}$ satisfies

$$
u_{A}\left(\Gamma^{j \mu}\right)_{B}^{A} k_{\mu}=0
$$

where $\Gamma^{j \mu}$ are the gamma matrices defined by the OPE 


$$
\psi^{j \mu}(z) S^{A}(w) \sim \frac{1}{\sqrt{2}} \frac{1}{\sqrt{z-w}}\left(\Gamma^{j \mu}\right)^{A}{ }_{B} S^{B} .
$$

The spin-field states in the $\mathrm{R}$ sector are bispinors that have bosonic statistics and that transform as a vector under the Lorentz group. The conditions (2.15) select a single state in the $\mathrm{R}$ sector. It is the $\mathrm{R}$ partner of the NS vacuum. Any other state in the $R$ sector, constructed from a spurious NS state, is itself spurious.

Therefore, the spectrum of the theory consists of two states.

A consequence of the simple structure of the open toy superstring is that the question of orientability does not arise at the level of the spectrum. However, the orientable and unorientable models may be distinguished at the loop level, as is discussed in Sec. IV.

Paton-Chan factors ${ }^{12}$ may also be attached to the ends of the open superstring. These factors provide a representation of an internal-symmetry group $G$. The specification of a state then also involves a matrix in that representation. In principle, the allowed groups and representations may be constrainted by requirements of unitarity and factorization of the amplitudes; see Sec. III.

\section{B. Closed superstring}

The basic closed toy superstring may also be constructed with two bosonic coordinates $X$ and $Y$ and two fermionic ones $\psi^{1}$ and $\psi^{2}$. The action is the same as that of the open toy superstring, Eqs. (2.1). The difference between the two models lies in the boundary conditions. For the bosonic coordinates, we assume periodic boundary conditions

$X^{\mu}\left(\sigma^{0}, \pi\right)=X^{\mu}\left(\sigma^{0}, 0\right), \quad Y^{\mu}\left(\sigma^{0}, \pi\right)=Y^{\mu}\left(\sigma^{0}, 0\right)$.

For the fermionic coordinates,

$$
\psi_{ \pm}^{j \mu}\left(\sigma^{0}, \pi\right)=R_{\phi, \pm}^{j k} \psi_{ \pm}^{k \mu}\left(\sigma^{0}, 0\right),
$$

where $R_{\phi, \pm} \in \mathrm{O}(2)$.

The bosonic coordinates split into sums of rightmoving parts, functions of $\sigma^{-}=\sigma^{0}-\sigma^{1}$ only, and leftmoving ones, functions of $\sigma^{+}=\sigma^{0}+\sigma^{1}$ only. Thus, for example, $X^{\mu}(\sigma)=X_{L}^{\mu}\left(\sigma^{+}\right)+X_{R}^{\mu}\left(\sigma^{-}\right)$. For the fermionic coordinates, $\psi_{+}^{j \mu}$ are functions of $\sigma^{+}$only, while $\psi^{j \mu}$ are functions of $\sigma^{-}$only.

The boundary conditions do not mix the left and the right movers. $R_{\phi,+}$ is the rotation for the left-moving fermions and $R_{\phi,-}$ is that for the right-moving ones. In each sector, according to the sign of the determinant of $R_{\phi}$, we have NS or $\mathrm{R}$ boundary conditions. There are therefore four sectors, which we denote (NS,NS), (NS,R), (R,NS), (R,R).

We first focus on the (NS,NS) sector, fixing the angle $\phi=\pi$ for both right and left movers. The string coordinates have normal-mode expansions

$$
\begin{aligned}
& X_{L}\left(\sigma^{+}\right)=\frac{1}{2} q^{X}+\frac{1}{2} p^{X} \sigma^{+}+\frac{i}{2} \sum_{n \neq 0} \frac{\widetilde{\alpha}_{n}^{X}}{n} e^{-2 i n \sigma^{+}}, \\
& Y_{L}\left(\sigma^{+}\right)=\frac{1}{2} q^{Y}+\frac{1}{2} p^{Y} \sigma^{+}+\frac{i}{2} \sum_{n \neq 0} \frac{\widetilde{\alpha}_{n}^{Y}}{n} e^{-2 i n \sigma^{+}}, \\
& \psi_{+}^{j}\left(\sigma^{+}\right)=\sum_{r \in \mathbb{Z}+1 / 2} \widetilde{b}_{r}^{j} e^{-2 i r \sigma^{+}} .
\end{aligned}
$$

Similar expressions hold for $X_{R}, Y_{R}$, and $\psi_{-}^{j}$, with $\widetilde{\alpha}^{X}$, $\widetilde{\alpha}^{Y}$, and $\widetilde{b}^{j}$ replaced by $\alpha^{X}, \alpha^{Y}$, and $b^{j}$, respectively.

Canonical quantization imposes the nonvanishing commutation relations

$$
\begin{aligned}
& {\left[\alpha_{m}^{X \mu}, \alpha_{n}^{X v}\right]=\left[\alpha_{m}^{Y \mu}, \alpha_{n}^{Y v}\right]=\left[\widetilde{\alpha}_{m}^{X \mu}, \widetilde{\alpha}_{n}^{X v}\right]=\left[\widetilde{\alpha}_{m}^{Y \mu}, \widetilde{\alpha}_{n}^{Y v}\right]} \\
& =m \delta_{m+n} \eta^{\mu v}, \\
& {\left[q^{X \mu}, p^{X v}\right]=\left[q^{Y \mu}, p^{Y v}\right]=i \eta^{\mu v},} \\
& \left\{b_{r}^{j \mu}, b_{s}^{k v}\right\}=\left\{\widetilde{b}_{r}^{j \mu}, \widetilde{b}_{s}^{k v}\right\}=\delta_{r+s} \eta^{\mu \nu} .
\end{aligned}
$$

In the conformal approach, we rotate to Euclidean time, $\sigma^{2}=i \sigma^{0}$, and introduce the conformal variables $z=e^{2\left(\sigma^{2}+i \sigma^{1}\right)}, \bar{z}=e^{2\left(\sigma^{2}-i \sigma^{1}\right)}$. The conformal fields are related to the string coordinates by

$$
\hat{X}=2 X, \quad \hat{Y}=2 Y, \quad \hat{\psi}_{+}^{j}=\frac{1}{\sqrt{z}} \psi_{+}^{j}, \quad \hat{\psi}_{-}^{j}=\frac{1}{\sqrt{z}} \psi_{-}^{j} .
$$

From now on we drop that caret over the conformal fields.

The action becomes

$S=\frac{1}{8 \pi \alpha^{\prime}} \int d z d \bar{z}\left(\partial X \cdot \bar{\partial} X+\partial Y \cdot \bar{\partial} Y+\psi_{+}^{j} \cdot \bar{\partial} \psi_{+}^{j}+\psi_{-}^{j} \cdot \partial \psi_{-}^{j}\right)$.

It is invariant under two independent sets of $N=2$ superconformal transformations, one for the right movers and one for the left movers. The associated currents have the structure (2.8) and generate two $N=2$ superconformal algebras of the form (2.9). The theory must therefore be supplemented with four pairs of ghost-antighost fields, $c$ $b, \gamma^{j}-\beta^{j}$, and $C-B$. All of them have both right and left movers. The analysis for the cancellation of the conformal anomaly is carried out independently in the two sectors and yields the critical dimensionality $D=2$.

According to the prescription that implements the projection, we want the $Y$ coordinates to be compactified on a torus of arbitrarily small radius $\epsilon$, defined as the quotient of $\mathbb{R}^{2}$ by a lattice $\Lambda_{0}$. This restricts the spectrum of the model to states with $p^{Y}=0$. The left and right movers can be treated independently:

$$
\begin{aligned}
& Y_{L}=q_{L}^{Y}+p_{L}^{Y} \sigma^{+}+\frac{i}{2} \sum_{n \neq 0} \frac{\widetilde{\alpha}_{n}^{Y}}{n} e^{-2 i n \sigma^{+}}, \\
& Y_{R}=q_{R}^{Y}+p_{R}^{Y} \sigma^{-}+\frac{i}{2} \sum_{n \neq 0} \frac{\alpha_{n}^{Y}}{n} e^{-2 i n \sigma^{-}},
\end{aligned}
$$

where $p_{L}^{Y}$ and $p_{R}^{Y}$ assume discrete values, multiples of $1 / \epsilon$. When $\epsilon$ tends to zero, only those states with $p_{L}^{Y}=p_{R}^{Y}=0$ remain at finite energies. Therefore, the spectrum contains these states only.

The physics of the closed toy superstring follows considerations similar to those of the previous section. Here, the map is from $S^{1}$ to $\mathbb{R}^{1}$. Taking into account the world-sheet supersymmetry, and since the center of mass involves both left and right coordinates, the model may be expected to contain four states. Next, we demonstrate this explicitly. 
The analysis of the physical spectrum parallels that of the open string. There is only one physical state in the (NS,NS) sector. The integrand of the BRST-invariant vertex operator associated with this state is

$$
\begin{aligned}
V^{\mathrm{NS}, \mathrm{NS}}(k)= & e^{i(k / 2) \cdot X_{L}} e^{i(k / 2) \cdot X_{R}} e^{-\phi_{+}^{1}-\phi_{+}^{2}-\phi_{-}^{1}-\phi_{-}^{2}} \\
& \times c(-1,-1 ;-1,-1),
\end{aligned}
$$

where $k$ is the momentum of the state and $c$ is the cocycle operator. We must require $k^{2}=0$ for this vertex operator to have the correct conformal dimension; therefore, the state is massless.

Similar conditions hold for the NS-R, R-NS, and R-R sectors. Integrands of the BRST-invariant vertex operators for states in these other sectors are obtained by using spin fields, e.g.,

$$
\begin{aligned}
V^{\mathrm{NS}, \mathrm{R}}(k, u)= & e^{i(k / 2) \cdot X_{L}} e^{-\phi_{+}^{1}-\phi_{+}^{2}} e^{i(k / 2) \cdot X_{R}} u_{A} S_{-}^{(A,-,-)} \\
& \times c(-1,-1 ; A,-,-)
\end{aligned}
$$

where $u_{A}$ satisfies the conditions (2.15).

The spectrum of the model therefore consists of four states.

\section{Heterotic strings}

Toy heterotic strings have added interest because their spectra are more complex. We consider here heterotic combinations of the $N=2$ closed string with the 26dimensional bosonic string. Note that our treatment of the $Y$ coordinates differs from that of Ref. 7 .

The string coordinates for this model are the following: the two-dimensional bosonic field $X=X_{L}+X_{R}$, the supersymmetric multiplet partners $Y_{R}$ and $\psi_{-}^{j}$ of $X_{R}$, and the internal bosonic left movers $X_{L}^{I}, I=1, \ldots, 24$. The action is

$$
\begin{aligned}
& S=\frac{1}{2 \pi \alpha^{\prime}} \int d \sigma^{+} d \sigma^{-}\left(\partial_{+} X \cdot \partial_{-} X+\partial_{+} Y_{R} \cdot \partial_{-} Y_{R}\right. \\
& \left.+\frac{i}{2} \psi^{j *} \cdot \partial_{+} \psi_{-}^{j}+\partial_{+} X_{L}^{I} \partial_{-} X_{L}^{I}\right) \text {. }
\end{aligned}
$$

For the left movers we assume periodic boundary conditions. As in the closed string, the boundary conditions for the right movers allow two sectors: NS and R. In the NS sector we set $\phi=\pi$. The string coordinates then have the normal-mode expansions

$$
\begin{aligned}
& X=q^{X}+p^{X} \sigma^{0}+\frac{i}{2} \sum_{n \neq 0}\left(\frac{\alpha_{n}^{X}}{n} e^{-2 i n \sigma^{-}}+\frac{\widetilde{\alpha}_{n}^{X}}{n} e^{-2 i n \sigma^{+}}\right) \\
& Y_{R}=q^{Y}+p^{Y} \sigma^{-}+\frac{i}{2} \sum_{n \neq 0} \frac{\alpha_{n}^{Y}}{n} e^{-2 i n \sigma^{-}}, \\
& \psi_{-}^{j}=\sum_{r \in \mathbb{Z}+1 / 2} b_{r}^{j} e^{-2 i r \sigma^{-}}, \\
& X_{L}^{I}=\widetilde{q}^{I+}+\widetilde{p}^{I} \sigma^{+}+\frac{i}{2} \sum_{n \neq 0} \frac{\widetilde{\alpha}_{n}^{I}}{n} e^{-2 i n \sigma^{+}} .
\end{aligned}
$$

Canonical quantization yields the following nonvanishing commutation relations:

$$
\begin{aligned}
& {\left[\alpha_{m}^{X \mu}, \alpha_{n}^{X v}\right]=\left[\alpha_{m}^{Y \mu}, \alpha_{n}^{Y v}\right]=\left[\widetilde{\alpha}_{m}^{X \mu}, \widetilde{\alpha}_{n}^{X v}\right]=m \delta_{m+n} \eta^{\mu \nu},} \\
& {\left[\widetilde{\alpha}_{m}^{I}, \widetilde{\alpha}_{n}^{J}\right]=m \delta_{m+n} \delta^{I J},} \\
& {\left[q^{X \mu}, p^{X v}\right]=i \eta^{\mu v}, \quad\left[q^{Y \mu}, p^{Y v}\right]=\frac{i}{2} \eta^{\mu \nu},} \\
& {\left[\widetilde{q}^{I}, \widetilde{p}^{J}\right]=\frac{i}{2} \delta^{I J}, \quad\left\{b_{r}^{j \mu}, b_{s}^{k v}\right\}=\delta_{r+s} \delta^{j k} \eta^{\mu \nu}}
\end{aligned}
$$

The factors of $\frac{1}{2}$ in the commutators of the zero modes of $Y_{R}$ and $X_{L}^{I}$ reflect the truncation of these coordinates to a single sector.

In the conformal approach the string coordinates are replaced by conformal fields as in Eq. (2.21). The action becomes

$$
\begin{gathered}
S=\frac{1}{8 \pi \alpha^{\prime}} \int d z d \bar{z}\left(\partial X \cdot \bar{\partial} X+\partial Y_{R} \cdot \bar{\partial} Y_{R}+\partial X_{L}^{I} \bar{\partial} X_{L}^{I}\right. \\
\left.+\psi_{-}^{j} \cdot \partial \psi_{-}^{j}\right) .
\end{gathered}
$$

From the commutation relations (2.28) we obtain the standard OPE for the conformal fields:

$$
\begin{aligned}
& X_{L}^{\mu}(z) X_{L}^{v}(\omega) \sim-\eta^{\mu v} \ln (z-\omega), \\
& X_{L}^{I}(z) X_{L}^{J}(\omega) \sim-\delta^{I J} \ln (z-\omega), \\
& X_{R}^{\mu}(\bar{z}) X_{R}^{v}(\bar{\omega}) \sim Y_{R}^{\mu}(\bar{z}) Y_{R}^{v}(\bar{\omega})=-\eta^{\mu v} \ln (\bar{z}-\bar{\omega}), \\
& \psi_{-}^{j \mu}(\bar{z}) \psi_{-}^{k v}(\bar{\omega}) \sim \delta^{j k} \eta^{\mu v} \frac{1}{\bar{z}-\bar{\omega}} .
\end{aligned}
$$

The action (2.29) is invariant under conformal transformations as well as under an $N=2$ supersymmetry and an $\mathrm{O}(2)$ symmetry among the right coordinates. Consequently, there is a set of conserved currents

$$
\begin{aligned}
& L=-\frac{1}{2}\left(\bar{\partial} X_{R} \cdot \bar{\partial} X_{R}+\bar{\partial} Y_{R} \cdot \bar{\partial} Y_{R}+\psi_{-}^{j} \cdot \bar{\partial} \psi_{-}^{j}\right), \\
& G^{j}=i\left(\psi_{-}^{j} \cdot \bar{\partial} X_{R}+\varepsilon^{j k} \psi_{-}^{k} \cdot \bar{\partial} Y_{R}\right), \quad T=-i \psi_{-}^{1} \cdot \psi_{-}^{2}
\end{aligned}
$$

that generate an $N=2$ superconformal algebra, and a current

$$
\widetilde{L}=-\frac{1}{2}\left(\partial X_{L} \cdot \partial X_{L}+\partial X_{L}^{I} \partial X_{L}^{I}\right)
$$

that generates a conformal algebra.

The theory must therefore be supplemented with ghosts $c, \gamma^{j}$, and $C$, and antighosts $b, \beta^{j}$, and $B$. The $c-b$ pair has both right and left movers; the others have only right movers. The analysis of the conformal dimension is carried out independently in each sector. As in the open string, the dimensionality in the right sector is fixed to be $D=2$. In the left sector, the cancellation of the conformal anomaly requires the number of internal coordinates to be 24 .

The physical states lie in the zero $Q$ - and $\widetilde{Q}$ cohomology class, where $Q$ and $\widetilde{Q}$ are the BRST operators for the superconformal ${ }^{24,25}$ and the conformal ${ }^{21}$ algebras, respectively. The mass of a physical state is given by

$$
\frac{1}{4}(\text { mass })^{2}=\frac{1}{2}\left(p^{Y}\right)^{2}+N+\frac{1}{2}\left(\widetilde{p}^{I}\right)^{2}+\widetilde{N}-1,
$$


where

$$
\begin{aligned}
& N=\sum_{n=1}^{\infty}\left(\alpha_{-n}^{X} \cdot \alpha_{n}^{X}+\alpha_{-n}^{Y} \cdot \alpha_{n}^{Y}\right)+\sum_{r=1 / 2}^{\infty} r b_{-r}^{j} \cdot b_{r}^{j}, \\
& \tilde{N}=\sum_{n=1}^{\infty}\left(\widetilde{\alpha}_{-n}^{X} \cdot \widetilde{\alpha}_{n}^{X}+\widetilde{\alpha}_{-n}^{I} \widetilde{\alpha}_{n}^{I}\right) .
\end{aligned}
$$

The level-matching condition is

$$
\frac{1}{2}\left(p^{Y}\right)^{2}+N=\frac{1}{2}\left(\widetilde{p}^{I}\right)^{2}+\widetilde{N}-1
$$

The internal coordinates $X_{L}^{I}$ take values in a compactified space. We take this to be a torus defined as the quotient of $\mathbb{R}^{24}$ by a lattice $\Lambda$. Following the philosophy outlined in the Introduction, we also take the $Y_{R}$ coordinates to be valued on a torus defined as the quotient of $\mathbb{R}^{2}$ by a lattice $\Lambda_{0}$. Note that $\left(\widetilde{p}^{I}\right)^{2}-\left(p^{Y}\right)^{2} \in 2 \mathbb{Z}$. The lattices and the radii of compactification are further restricted by modular invariance, as discussed in Sec. IV.

An analysis similar to that for the open string shows that at the massless level in the right sector there are two states, for which $p^{Y}=0$ and $N=0$. Therefore, in the left sector we must consider only states for which $\frac{1}{2}\left(\widetilde{p}^{I}\right)^{2}+\widetilde{N}-1=0$. This condition is satisfied if either $\left(\widetilde{p}^{I}\right)^{2}=2$ and $\widetilde{N}=0$ or $\left(\widetilde{p}^{I}\right)^{2}=0$ and $\widetilde{N}=1$. In the former case there are winding excitations (charged states) $\left|\widetilde{p}^{I}\right\rangle^{L} \otimes|0\rangle^{R}$; in the latter there are oscillator excitations (neutral states) $\widetilde{\alpha}_{-1}^{I}|0\rangle^{L} \otimes|0\rangle^{R}$. The potentially physical state with a two-dimensional excitation $\xi \cdot \widetilde{\alpha}_{-1}^{X}|0\rangle^{L} \otimes|0\rangle^{R}$ is spurious and need not be considered. For example, if the lattice $\Lambda$ is $D_{24}$ there are $2^{2} \times 24 \times 23 / 2=1104$ charged states and 24 neutral ones, for a total of 1128 states.

The vertex operators associated with these states are

$$
\begin{aligned}
& V^{c}(k, h)=e^{i h^{I} X_{L}^{I}} e^{i(k / 2) \cdot X_{L}} e^{i(k / 2) \cdot X_{R}} c(h), \\
& V^{n}(k, \zeta)=\zeta^{I} \dot{X}_{L}^{I} e^{i(k / 2) \cdot X_{L}} e^{i(k / 2) \cdot X_{R}},
\end{aligned}
$$

where $k$ is the two-dimensional momentum, $h$ is the 24dimensional one, $\zeta$ is a polarization vector, and $c(h)$ is the cocycle operator. The correct conformal dimension is ensured by the conditions $k^{2}=0$ and $h^{2}=2$.

Alternatively, the internal coordinates can be described in the fermionic representation. The 24 fields $X_{L}^{I}$ are replaced by 48 fermions

$$
\psi_{+}^{j}=\sum_{r \in \mathbb{Z}+1 / 2} \widetilde{b}_{r}^{j} e^{-2 i r \sigma^{+}}
$$

A massless state has the form $T^{j k} \widetilde{b}_{-1 / 2}^{j} \widetilde{b}_{-1 / 2}^{k}|0\rangle$, where $T^{j k}$ is an antisymmetric matrix describing the polarization of the state. For example, in the case of $D_{24}$ we must take all possible antisymmetric matrices, so that there are
$48 \times 47 / 2=1128$ independent states. Other lattices involve suitable projections and recombinations typically resulting in a different number of states, much as for the usual fermionic representation of the $E_{8} \times E_{8}$ heterotic string.

The vertex operator in the fermionic representation is

$$
V(k, T)=\sum_{m} T^{j k}\left(Q^{j k}\right)_{m} e^{i(k / 2) \cdot X_{L}} e^{i(k / 2) \cdot X_{R}},
$$

where the $\left(Q^{j k}\right)_{m}$ are the moments of the bilinear fermionic current $Q^{j k}=\frac{1}{2} \psi_{+}^{j} \psi_{+}^{k}$ forming a representation of the affine so(48) algebra.

\section{TREE AMPLITUDES}

In this section we obtain tree-level scattering amplitudes for the toy superstrings presented in Sec. II. For definiteness we treat four-point amplitudes, but the computations can be generalized to $n$ points. Note that at the tree level the one-, two-, and three-point amplitudes are trivial: the one-point amplitudes vanish since a state with nonzero momentum cannot disappear into the vacuum; the two-point amplitudes describe the free propagation of the state; while the vanishing of the three-point amplitudes implies the absence of three-point vertices at the string level.

For simplicity, in what follows we omit from the expressions for amplitudes the delta functions ensuring conservation of momenta. We also set the coupling constants $g$ and $\kappa$ to one.

\section{A. Open superstring}

We begin by presenting the calculation of the tree-level amplitude for four external NS states in the open toysuperstring model. The vertex for the open-superstring state in the $F_{1}$ formalism is ${ }^{33}$

$$
W(k, z)=: e^{i k \cdot X(z)}: \text {, }
$$

where $z=e^{i \sigma^{+}}, k^{2}=0$, and where $\sigma^{1}$ has been extended from $-\pi$ to 0 with the bosonic coordinates defined symmetrically. The vertex in the $F_{2}$ formalism $^{34}$ is

$$
\begin{aligned}
V(k, z) & =z^{-t-s}\left\{G_{t}^{2},\left[G_{s}^{1}, W(k, z)\right]\right\} \\
& =-z^{-t-s}\left\{G_{s}^{1},\left[G_{t}^{2}, W(k, z)\right]\right\} \\
& =\left[k \cdot \dot{Y}(z)+2 k \cdot \psi^{1}(z) k \cdot \psi^{2}(z)\right]: e^{i k \cdot X(z)}:,
\end{aligned}
$$

where the $G_{t}^{j}$ are the moments of the supersymmetry currents $G^{j}$ and where the overdot denotes a derivative with respect to $\sigma^{0}$.

The four-point tree-level amplitude may be computed using coherent-state methods. The result for the $u$ channel is ${ }^{1}$ 


$$
\begin{aligned}
A_{4 \mathrm{NS}, u}^{\text {open }} & =\int_{0}^{1} \frac{d z}{z}\left\langle-k_{1}\left|V\left(k_{2}, 1\right) V\left(k_{3}, z\right)\right| k_{4}\right\rangle \\
& =\int \frac{d z}{z}\left\langle-k_{1}\left|\left[k_{2} \cdot \dot{Y}(1)+2 k_{2} \cdot \psi^{1}(1) k_{2} \cdot \psi^{2}(1)\right]: e^{i k_{2} \cdot X(1)}:\left[k_{3} \cdot \dot{Y}(z)+2 k_{3} \cdot \psi^{2}(z) k_{3} \cdot \psi^{1}(z)\right]: e^{i k_{3} \cdot X(z)}:\right| k_{4}\right\rangle \\
& =\int \frac{d z}{z} z^{k_{3} \cdot k_{4}}(1-z)^{k_{2} \cdot k_{3}} k_{2} \cdot k_{3}\left(1-k_{2} \cdot k_{3}\right) \frac{z}{(1-z)^{2}}=-\frac{\Gamma\left[1-\frac{s}{2}\right] \Gamma\left(1-\frac{t}{2}\right)}{\Gamma\left[\frac{u}{2}\right]}
\end{aligned}
$$

This amplitude has cyclic symmetry.

The same result is readily obtained using conformal methods. The amplitude for four scalar states involves the vertices $c V_{(-1,-1)}^{(\mathrm{NS})}(k), \int d z V_{(-1,-1)}^{(\mathrm{NS})}(k)$ and their BRST transforms $c V_{(0,0)}^{(\mathrm{NS})}(k), \int d z V_{(0,0)}^{(\mathrm{NS})}(k)$. Here, $V_{(-1,-1)}^{(\mathrm{NS})}(k)$ is given by Eq. (2.11). A BRST transform is obtained via a $\xi$ manipulation ${ }^{21}$ and is the conformal equivalent of a vertex in the $F_{2}$ formalism. Thus, we find

$$
c V_{(0,0)}^{(\mathrm{NS})}(k)=\left[Q, \xi^{1}\left[Q, \xi^{2} c V_{(-1,-1)}^{(\mathrm{NS})}(k)\right]\right]=c\left(i k \cdot \partial Y+k \cdot \psi^{1} k \cdot \psi^{2}\right) e^{i k \cdot X}+\text { ghosts },
$$

with an analogous expression for $\int d z V_{(0,0)}^{(\mathrm{NS})}(k)$. Here, $Q$ is the BRST operator for the $N=2$ superconformal algebra (2.9) (Refs. 24 and 25). The amplitude for four external scalar states is

$$
\begin{aligned}
A_{4 \mathrm{NS}, u}^{\mathrm{open}} & =\left\langle c e^{-\phi_{1}-\phi_{2}} e^{i k_{1} \cdot X} c\left(i k_{2} \cdot \partial Y+k_{2} \cdot \psi^{1} k_{2} \cdot \psi^{2}\right) e^{i k_{2} \cdot X} \int d z\left(i k_{3} \cdot \partial Y-k_{3} \cdot \psi^{1} k_{3} \cdot \psi^{2}\right) e^{i k_{3} \cdot X} c e^{-\phi_{1}-\phi_{2}} e^{i k_{4} \cdot X}\right\rangle \\
& =-\frac{\Gamma\left(1-\frac{s}{2}\right) \Gamma\left(1-\frac{t}{2}\right)}{\Gamma\left(\frac{u}{2}\right)} .
\end{aligned}
$$

Conformal techniques allow also the computation of amplitudes involving an even number of spin-field states. Amplitudes with an odd number of spin-field states vanish due to statistics. To evaluate four-point amplitudes, we need the BRST-invariant spin-field vertices $c V_{(-,-)}^{(\mathbf{R})}(k)$ and $\int d z V_{(-,-)}^{(\mathbf{R})}(k)$ obtained from Eq. (2.14). Taking into account the physical conditions (2.15), the polarization of the spin-field vertices is $u_{ \pm \pm}=\sqrt{i / 2}\left(k_{1} \pm k_{0}\right)$. The amplitudes with four spin-field states and with two scalar states and two spin-field states evaluated in the $u$ channel are

$$
\begin{aligned}
A_{4 \mathrm{R}, u}^{\text {open }} & =u_{A_{1}} u_{A_{2}} u_{A_{3}} u_{A_{4}}\left\langle c S^{\left(A_{1},-,-\right)} e^{i k_{1} \cdot X} c S^{\left(A_{2},-,-\right)} e^{i k_{2} \cdot X} \int S^{\left(A_{3},-,-\right)} e^{i k_{3} \cdot X} c S^{\left(A_{4},-,-\right)} e^{i k_{4} \cdot X}\right\rangle \\
& =k_{2} \cdot k_{3} k_{3} \cdot k_{4} k_{4} \cdot k_{2} \frac{\Gamma\left(k_{3} \cdot k_{4}\right) \Gamma\left(k_{2} \cdot k_{3}\right)}{\Gamma\left(1+k_{3} \cdot k_{4}+k_{2} \cdot k_{3}\right)}=-\frac{\Gamma\left(1-\frac{t}{2}\right) \Gamma\left(1-\frac{s}{2}\right)}{\Gamma\left(\frac{u}{2}\right)}
\end{aligned}
$$

and

$$
\begin{aligned}
& A_{2 \mathrm{NS} 2 \mathrm{R}, u}^{\mathrm{open}}=u_{A_{2}} u_{A_{3}}\left\langle c e^{-\phi_{1}-\phi_{2}} e^{i k_{1} \cdot X} c S^{\left(A_{2},-,-\right)} e^{i k_{2} \cdot X} \int S^{\left(A_{3},-,-\right)} e^{i k_{3} \cdot X} c\left(i k \cdot \partial Y-k_{4} \cdot \psi^{1} k_{4} \cdot \psi^{2}\right) e^{i k_{4} \cdot X}\right\rangle \\
& =k_{2} \cdot k_{4} k_{3} \cdot k_{4} \frac{\Gamma\left(k_{3} \cdot k_{4}\right) \Gamma\left(1+k_{2} \cdot k_{3}\right)}{\Gamma\left(1+k_{2} \cdot k_{3}+k_{3} \cdot k_{4}\right)}=-\frac{\Gamma\left[1-\frac{s}{2}\right] \Gamma\left(1-\frac{t}{2}\right)}{\Gamma\left(\frac{u}{2}\right)} .
\end{aligned}
$$

These coincide with the four-scalar amplitude, Eq. (3.3). This might have been expected on the basis of world-sheet supersymmetry; nevertheless, it is a nontrivial result that arises from appropriate recombinations of terms in the expressions for the amplitudes.

The full amplitude, invariant under all permutations of external states, is found by summing over inequivalent channels: 


$$
A_{4}^{\mathrm{open}}=-\frac{1}{2}\left(\frac{\Gamma\left(1-\frac{s}{2}\right) \Gamma\left(1-\frac{t}{2}\right)}{\Gamma\left(\frac{u}{2}\right)}+\frac{\Gamma\left(1-\frac{t}{2}\right) \Gamma\left(1-\frac{u}{2}\right)}{\Gamma\left(\frac{s}{2}\right)}+\frac{\Gamma\left(1-\frac{u}{2}\right) \Gamma\left(1-\frac{s}{2}\right)}{\Gamma\left(\frac{t}{2}\right)}\right) .
$$

Since $D=2, A_{4}^{\text {open }}$ has no pole and is identically zero. This may be viewed as a consequence of the vanishing of the three-point amplitudes. The factorization of the four-point amplitude for an intermediate state on mass shell is trivial because the three-point amplitudes that would appear in the residue of the singularity are zero.

Note that when Paton-Chan factors ${ }^{12}$ are included, the four-point amplitude may not vanish in general. Furthermore, constraints on the possible assignment of Paton-Chan factors may arise from factorization of the six-point or higher-point amplitudes.

\section{B. Closed superstring}

For the closed toy superstring, the vertex in the (NS,NS) sector is

$$
V(k)=4 V_{R}\left(\frac{k}{2}, 1\right) V_{L}\left(\frac{k}{2}, 1\right),
$$

where $V_{R}$ and $V_{L}$ have the form (3.2).

In the computation of the amplitude $A=\left\langle V_{1} \Delta V_{2} \Delta V_{3} \Delta V_{4}\right\rangle$, the propagator is replaced by

$$
\Delta=\frac{-1}{4 \pi} \int_{|z| \leq 1} \frac{d z d \bar{z}}{2 i z \bar{z}} \bar{z}^{L_{0}} \bar{L}^{\tilde{L}_{0}}
$$

to ensure that only those states satisfying the constraint $L_{0}-\widetilde{L}_{0}=0$ propagate.

The integrand in the amplitude factorizes into the product of two open-string integrands. However, the region of integration is different for the closed superstring, being over the interior of the world sheet rather than over its boundary. Furthermore, the region of integration is extended from the unit disk to the whole complex plane to ensure summation over the possible radial orderings of the external states.

The amplitude with four scalar states is

$$
\begin{aligned}
A_{4 \mathrm{NS}}^{\text {closed }} & =\frac{16}{4 \pi} \int \frac{d^{2} z}{z \bar{z}}\left\langle-\frac{k_{1}}{2}\left|V_{R}\left(\frac{k_{2}}{2}, 1\right) V_{R}\left(\frac{k_{3}}{2}, \bar{z}\right)\right| \frac{k_{4}}{2}\right\rangle\left\langle-\frac{k_{1}}{2}\left|V_{L}\left(\frac{k_{2}}{2}, 1\right) V_{L}\left(\frac{k_{3}}{2}, z\right)\right| \frac{k_{4}}{2}\right\rangle \\
& \left.=\frac{4}{\pi} \int d^{2} z|z|^{\left(k_{3} \cdot k_{4}\right) / 2}|1-z|^{\left(k_{2} \cdot k_{3}\right) / 2-4}\left(\frac{k_{2} \cdot k_{3}}{4}\right)^{2} \mid 1-\frac{k_{2} \cdot k_{3}}{4}\right)^{2} \\
& =4 \frac{\Gamma\left(1-\frac{s}{8}\right) \Gamma\left(1-\frac{t}{8}\right) \Gamma\left(1-\frac{u}{8}\right)}{\Gamma\left(\frac{s}{8}\right) \Gamma\left(\frac{t}{8}\right) \Gamma\left(\frac{u}{8}\right)} .
\end{aligned}
$$

This amplitude is invariant under any interchange of the external particles, as expected. Like the open-superstring amplitude, the closed-superstring amplitude does not display any pole structure. This can again be understood as a consequence of the vanishing of the three-point amplitudes.

Using conformal techniques, one can compute this amplitude and others involving also spin-field states. We have explicitly verified that all nontrivial four-point amplitudes give the same result, Eq. (3.11). This might be anticipated on the basis of world-sheet supersymmetry.

\section{Heterotic strings}

For massless states of the toy heterotic strings, the vertex has the form (3.9) with $V_{R}$ an open-superstring vertex and $V_{L}$ a suitable vertex for the left-moving sector. by

Taking into account the level-matching condition for propagating states, the propagator $\frac{1}{2}\left(L_{0}+\widetilde{L}_{0}-1\right)^{-1}$ is replaced

$$
\Delta=\frac{1}{4 \pi} \int \frac{d^{2} z}{z \bar{z}} \bar{z}^{L_{0}} z^{\tilde{L}_{0}-1}
$$


The factor -1 arises from the shift in the mass formula.

We consider in detail amplitudes involving four external scalar states. In the bosonic description, neutral and charged internal-symmetry states are associated with distinct vertices as in (2.36). In this approach, there are different possible four-point tree-level amplitudes for different choices of external states.

The amplitude for four charged states with internal momentum $h_{r}$ is

$$
\begin{aligned}
A_{h_{1}, h_{2}, h_{3}, h_{4}}^{\text {het }} & =(-)^{U / 2} \epsilon_{23} \epsilon_{34} \frac{1}{4} \frac{\Gamma\left(1-\frac{s}{8}\right) \Gamma\left(1-\frac{t}{8}\right) \Gamma\left(1-\frac{u}{8}\right)}{\Gamma\left(2+\frac{s}{8}-\frac{S}{2}\right) \Gamma\left(2+\frac{t}{8}-\frac{T}{2}\right) \Gamma\left(2+\frac{u}{8}-\frac{U}{2}\right)} \\
& \equiv(-)^{U / 2} \epsilon_{23} \epsilon_{24} \epsilon_{34} \frac{1}{4} \frac{\Gamma\left(-1-\frac{s}{8}+\frac{S}{2}\right) \Gamma\left(-1-\frac{t}{8}+\frac{T}{2}\right) \Gamma\left(-1-\frac{u}{8}+\frac{U}{2}\right)}{\Gamma\left(\frac{s}{8}\right) \Gamma\left(\frac{t}{8}\right) \Gamma\left(\frac{u}{8}\right)},
\end{aligned}
$$

where $\epsilon_{i j}=\epsilon\left(h_{i}, h_{j}\right)$ are the cocycle factors, and $S=\left(h_{3}+h_{4}\right)^{2}=4+2 h_{3} \cdot h_{4}$, etc. The amplitude with one neutral state is

$$
A_{h_{1}, \xi_{2}, h_{3}, h_{4}}^{\text {het }}=-\frac{1}{16} \epsilon_{13} \epsilon_{14} \epsilon_{34} \gamma\left(k_{2} \cdot k_{4} \xi_{2} \cdot h_{3}-k_{2} \cdot k_{3} \xi_{2} \cdot h_{4}\right) \text {, }
$$

where

$$
\gamma=\frac{\Gamma\left(-\frac{s}{8}\right) \Gamma\left(-\frac{t}{8}\right) \Gamma\left(-\frac{u}{8}\right)}{\Gamma\left(\frac{s}{8}\right) \Gamma\left(\frac{t}{8}\right) \Gamma\left(\frac{u}{8}\right)},
$$

and $\zeta_{2}$ is the polarization of the neutral state. The amplitude with two neutral states is

$$
\begin{aligned}
A_{h_{1}, \zeta_{2}, \xi_{3}, h_{4}}^{\text {het }}=\frac{1}{16} \epsilon_{14} \gamma & \zeta_{3} \cdot h_{4} \zeta_{2} \cdot h_{4} k_{2} \cdot k_{3} \\
& \left.+\zeta_{2} \cdot \zeta_{3} \frac{k_{2} \cdot k_{4} k_{3} \cdot k_{4}}{4-k_{2} \cdot k_{3}}\right] .
\end{aligned}
$$

The amplitude with three neutral states vanishes due to lattice-charge conservation. The amplitude with four neutral states is

$$
A_{\zeta_{1}, \zeta_{2}, \zeta_{3}, \zeta_{4}}^{\text {het }}=\frac{1}{16} \gamma\left[\zeta_{3} \cdot \zeta_{4} \xi_{1} \cdot \zeta_{2} \frac{k_{2} \cdot k_{4} k_{2} \cdot k_{3}}{4-k_{3} \cdot k_{4}}+\text { cyclic }\right] \text {. }
$$

All four amplitudes (3.13), (3.14), (3.16), and (3.17) are invariant under permutation of the external states.

These amplitudes are unified in the fermionic description. The vertex $V(k, T)$ is given by Eq. (2.38). The contribution of the internal coordinates to the integrand of the four-point amplitude is

$$
\left\langle 0\left|T_{1} Q_{1}\left(\sum_{m} T_{2} Q_{m}\right) z^{\tilde{L}_{0}-2}\left(\sum_{n} T_{3} Q_{n}\right) T_{4} Q_{-1}\right| 0\right\rangle .
$$

Using the properties of the $Q_{m}$ (Ref. 11),

$$
\begin{aligned}
& Q_{m}|0\rangle=0, \quad m \geq 0 ; \quad z^{n} Q_{m}=Q_{m} z^{n-m}, \\
& {\left[T_{i} Q_{m}, T_{j} Q_{n}\right]=\left[T_{i}, T_{j}\right] Q_{m+n}+\frac{m}{2} \delta_{m+n} \operatorname{tr}\left(T_{i} T_{j}\right),}
\end{aligned}
$$

the contribution (3.18) may be determined. The contribution from the $X^{\mu}$ and the right-moving sector is

$$
\begin{aligned}
& \frac{1}{4 \pi} \frac{1}{\bar{z}} \frac{\bar{z}}{(1-\bar{z})^{2}} \frac{k_{2} \cdot k_{3}}{4} {\left[1-\frac{k_{2} \cdot k_{3}}{4}\right] } \\
& \times|z|^{\left(k_{3} \cdot k_{4}\right) / 2}|1-z|^{\left(k_{2} \cdot k_{3}\right) / 2} .
\end{aligned}
$$

Integrating over the complex plane, the final result is

$$
\begin{aligned}
A_{4}^{\text {het }}=-4 & \frac{\Gamma\left(1-\frac{s}{8}\right) \Gamma\left(1-\frac{t}{8}\right) \Gamma\left(1-\frac{u}{8}\right)}{\Gamma\left(\frac{s}{8}\right) \Gamma\left(\frac{t}{8}\right) \Gamma\left(\frac{u}{8}\right)} \\
& \times\left(\frac{\operatorname{tr}\left(T_{1} T_{2}\right) \operatorname{tr}\left(T_{3} T_{4}\right)}{s(8+s)}\right. \\
& \left.-2 \frac{\operatorname{tr}\left(T_{1} T_{3} T_{2} T_{4}+T_{1} T_{4} T_{2} T_{3}\right)}{t u}+\text { cyclic }\right),
\end{aligned}
$$

where $T_{r}$ are the polarization matrices for the external states.

The same result is found using the conformal approach. Scattering amplitudes with spin-field states may similarly be determined via conformal techniques. As in the cases of the open and closed toy superstrings, these amplitudes coincide with (3.21).

The amplitude (3.21) exhibits duality; i.e., it is invariant under interchange of the external states. It does not vanish identically. Nontrivial scattering among the string states is possible. 
The structure of this amplitude is similar to the corresponding amplitude for the ten-dimensional heterotic string. ${ }^{11}$ The first term in the parenthesis can be associated in the 26-dimensional sector with a disconnected diagram as it arises from $\left\langle 0\left|V_{1} V_{2}\right| 0\right\rangle\left\langle 0\left|V_{3} V_{4}\right| 0\right\rangle$ in the evaluation of the amplitude. The second term can be interpreted as the exchange of an intermediate state between the initial state containing the particles 1,2 and the final state containing 3,4 . The absence of a pole associated with the intermediate state cannot be imputed to the 26-dimensional sector of the theory. Indeed, this sector allows for a three-point vertex proportional to $\operatorname{tr}\left(T_{1} T_{2} T_{3}\right)$. However, since

$$
\begin{aligned}
\left\langlec e ^ { - \phi _ { 1 } - \phi _ { 1 } } e ^ { i k _ { 1 } \cdot X } \cdot c \left( i k_{2} \cdot \partial Y\right.\right. & \left.-k_{2} \cdot \psi^{1} k_{2} \cdot \psi^{2}\right) e^{i k_{2} \cdot X} \\
& \left.\times c e^{-\phi_{1}-\phi_{2}} e^{i k_{3} \cdot X}\right\rangle=0,
\end{aligned}
$$

the two-dimensional sector causes the three-point vertex to vanish.

\section{ONE-LOOP AMPLITUDES}

In this section, we present one-loop four-point scalarstate amplitudes for the toy superstrings of Sec. II. We begin with some remarks about the lower-point amplitudes, all of which vanish to this order.

At the one-loop level, the one-point amplitude contains a factor $\langle\Delta V\rangle$. In the open and the heterotic toy superstrings this vanishes because the vertex contains $\left(k \cdot \dot{Y}-2 k \cdot \psi^{1} k \cdot \psi^{2}\right)$. In the closed toy superstring, the only nonvanishing contribution could come from the zero modes of $Y$, which enter both $V_{L}$ and $V_{R}$; however, this vanishes on-shell since $k^{2}=0$. The vanishing of the onepoint amplitude corresponds to a zero one-loop contribution to the vacuum expectation values of the string states.

In all the models, the two-point amplitude is proportional to $k_{1} \cdot k_{2}$ due to the trace over $Y$ and $\psi^{1} \psi^{2}$. Since $k_{1}=-k_{2}$ and $k_{j}^{2}=0$, the amplitudes vanish. The propagator receives no one-loop corrections.

The three-point amplitudes for the open and the heterotic toy superstrings could have nonzero contributions only from the trace involving the zero modes of $Y$. However, the dynamics of the process excludes this possibility. For the closed toy superstring, the same analysis is slightly more involved because the zero modes of $Y$ are common to the left and right vertices. Nevertheless, the result holds. Thus, the three-point vertices of these toy modes remain zero at one-loop order.

\section{A. Open superstring}

For the open toy superstring, the simplest four-point contribution is the planar loop. This involves the trace of the product of the external vertices with propagators acting between adjacent vertices.

The contribution from the bosonic coordinate $X$ has a form similar to that of the 26-dimensional bosonic string:

$$
\begin{aligned}
& \prod_{n=1}^{\infty}\left(1-\omega^{n}\right)^{-D} \prod_{n=1}^{\infty} \exp \left[-\frac{1}{n} \sum_{r<s} k_{r} \cdot k_{s} \frac{c_{s r}^{n}+\omega^{n} c_{s r}^{-n}}{1-\omega^{n}}\right] \\
& \times\left(\frac{-2 \pi}{\ln \omega}\right]^{D / 2} \prod_{r<s}\left[c_{s r}^{-1 / 2} \exp \left[\frac{\ln ^{2} c_{s r}}{2 \ln \omega}\right]\right]^{k_{r} \cdot k_{s}},
\end{aligned}
$$

where $k_{r}$ are the external momenta and $D=2$. Here, $r, s=1, \ldots, 4$ denote the external states and $\omega=x_{1} x_{2} x_{3} x_{4}, c_{s r}=x_{r+1} \ldots x_{s}$. The result can be expressed in a more compact form with the function ${ }^{35}$

$$
\psi(c, \omega)=-2 \pi i \exp \left[\frac{i \pi v^{2}}{\tau}\right] \frac{\theta_{1}(v \mid \tau)}{\theta_{1}^{\prime}(0 \mid \tau)}
$$

Here, $\theta_{1}$ is Jacobi's theta function, ${ }^{36}$ and the variables $v=v_{s r}$ and $\tau$ are related to $c=c_{s r}$ and $\omega$ by $\nu=\ln c / 2 \pi i$, $\tau=\ln \omega / 2 \pi i$. For simplicity, we write $\psi_{r s}$ for $\psi\left(c_{s r}, \omega\right)$. In terms of these quantities, Eq. (4.1) becomes

$$
\prod_{n=1}^{\infty}\left(1-\omega^{n}\right)^{-D}\left(\frac{-2 \pi}{\ln \omega}\right)^{D / 2} \exp \left(\sum_{r<s} k_{r} \cdot k_{s} \ln \psi_{r s}\right) .
$$

The contributions to the amplitude for the planar loop due to $\dot{Y}$ and $\psi^{1}, \psi^{2}$ can be split into three groups: one for the traces of two $\dot{Y}$ and two $\psi^{1} \psi^{2}$, another for the trace of four $\psi^{1} \psi^{2}$, and the last for the trace of four $\dot{Y}$. These traces may be evaluated by exponentiating the various terms and using coherent-state methods. At the end of the calculation, only the terms linear in all the polarizations are kept.

Particular care is important in dealing with the zero modes of $Y$. Although these modes do not enter the computation of the tree-level diagrams, they are essential for one- and higher-loop amplitudes. They may be treated as follows, using the prescription for the projection discussed in the Introduction. The absence of $q^{Y}$ in the vertex is due to the translational invariance in the $Y$ directions. We take $Y$ to be defined on a torus of arbitrarily small radius $\epsilon$. The momentum $p^{Y}$ therefore take values on a lattice and is quantized in multiples of $1 / \epsilon$. The integral over $p^{Y}$ is then replaced by a sum over the lattice points. In the evaluation of the loop amplitude, we must take moments of $\omega^{L_{0}}$ with respect to $p^{Y}$. We therefore need to consider a sum of the form

$$
\begin{aligned}
\sum_{m=-\infty}^{\infty}\left(\frac{m^{2}}{4 \epsilon^{2}}\right]^{k} \exp \left[\frac{1}{2} \frac{m^{2}}{4 \epsilon^{2}} \ln \omega\right] & =\left[\frac{1}{2}\right]^{k}\left[\left(\partial_{A}\right)^{k} \sum_{m} \exp \left[-\frac{1}{2} A \frac{m^{2}}{\epsilon^{2}}\right]\right]_{A=-(\ln \omega) / 4} \\
& =\sqrt{2 \pi} \frac{(2 k-1) ! !}{(-\ln \omega)^{k}}+O(\epsilon) .
\end{aligned}
$$


This is just the result one would get if $Y$ were not compactified, except for a factor $(-\ln |\omega| / 2 \pi)^{D / 2}$. Thus, the net effect of taking the radius of compactification $\epsilon$ to zero is that we can perform the Gaussian integration over $p^{Y}$ and afterwards replace the factor $(-2 \pi / \ln |\omega|)^{D / 2}$ by one.

The result of the trace over products of $\dot{Y}$ is

$$
\begin{array}{r}
\prod_{n=1}^{\infty}\left(1-\omega^{n}\right)^{-D} \exp \left[\sum _ { r < s } \hat { k } _ { r } \cdot \hat { k } _ { s } \left[\sum_{n=1}^{\infty} \frac{n}{1-\omega^{n}}\left(c_{s r}^{n}+\omega^{n} / c_{s r}^{n}\right)\right.\right. \\
\left.\left.-\frac{1}{\ln \omega}\right]\right] .
\end{array}
$$

The first factor is the bosonic partition function. Of the two terms in the exponential, the first is due to the trace over the oscillator modes, while the second is the result of the integration over $p^{Y}$. It is understood that one has to expand the exponential in power series, keep only the terms linear in all the $\hat{k}_{r}$, and set $\hat{k}_{r}=k_{r}$. This can be accomplished introducing an integration over Grassmann variables $\vartheta_{r}$ and $\varphi_{r}$. Equation (4.5) then becomes

$$
\begin{aligned}
\prod_{n=1}^{\infty}\left(1-\omega^{n}\right)^{-D} \prod_{r} & {\left[\int d \vartheta_{r} d \varphi_{r}\right) } \\
& \times \exp \left(\sum_{r<s} k_{r} \cdot k_{s} \vartheta_{r} \varphi_{r} \vartheta_{s} \varphi_{s} \Omega_{r s}\right),
\end{aligned}
$$

where

$$
\begin{aligned}
\Omega(c, \omega) & =\frac{-1}{\ln \omega}+\sum_{n=1}^{\infty} \frac{n}{1-\omega^{n}}\left(c^{n}+\omega^{n} / c^{n}\right) \\
& =-c \partial_{c}\left[c \partial_{c} \ln \psi(c, \omega)\right] .
\end{aligned}
$$

In addition to the fermionic partition function $\prod_{r=1 / 2}^{\infty}\left(1-\omega^{r}\right)^{2 D}$, the trace over the fermionic coordinates gives

$$
\text { (士) }\left[\exp \left(\sum_{r<s} \hat{k}_{r} \cdot \hat{k}_{s} \sum_{t=1 / 2}^{\infty}\left(c_{s r}^{t}-\omega^{t} / c_{s r}^{t}\right)\right)\right]^{2}
$$

where the sign is negative for a two-fermion trace or for a four-fermion one in which 1 is paired with 3 and 2 with 4 . This expression can also be written as an integral over Grassmann variables:

$$
\prod_{r}\left[\int d \vartheta_{r} d \varphi_{r}\right] \exp \left(\sum_{r<s} k_{r} \cdot k_{s}\left(\vartheta_{r} \vartheta_{s}+\varphi_{r} \varphi_{s}\right) \Psi_{r s}\right],
$$

where

$$
\Psi(c, \omega)=\sum_{t=1 / 2}^{\infty} \frac{1}{1-\omega^{t}}\left(c^{t}-\omega^{t} / c^{t}\right)
$$

is the two-point fermionic correlation function for the annulus. It can be expressed as

$$
\begin{aligned}
& \psi(c, \omega)=\eta(c, \omega)-\eta(\sqrt{c}, \sqrt{\omega}), \\
& \eta(c, \omega)=c \partial_{c} \ln \psi(c, \omega) .
\end{aligned}
$$

Assembling the contributions of the three groups yields

$$
I \prod_{n=1}^{\infty}\left(1-\omega^{n}\right)^{-D} \prod_{r=1 / 2}^{\infty}\left(1-\omega^{r}\right)^{2 D},
$$

where

$$
I=\prod_{i=1}^{4}\left[\int d \vartheta_{i} d \varphi_{i}\right] \exp \left(\sum_{r<s} k_{r} \cdot k_{s}\left(\Psi_{r s} \vartheta_{r} \vartheta_{s}+\Psi_{r s} \varphi_{r} \varphi_{s}-\Omega_{r s} \vartheta_{r} \vartheta_{s} \varphi_{r} \varphi_{s}\right)\right]
$$

There is a further contribution from the trace over the two bosonic- and two fermionic-ghost excitations. This cancels the partition functions in Eqs. (4.3) and (4.12).

The resulting amplitude is

$$
\begin{aligned}
A_{P}^{(\mathrm{NS})=} \prod_{i=1}^{4}\left(\int d \vartheta_{i} d \varphi_{i}\right) \int_{0}^{1} \frac{d \omega}{\omega} \prod_{r=1}^{3} \int_{0}^{1} \frac{d \rho_{r}}{\rho_{r}} \theta\left(\rho_{r}-\rho_{r+1}\right) \frac{-2 \pi}{\ln \omega} \\
\\
\quad \times \exp \left(\sum_{r<s} k_{r} \cdot k_{s}\left[\ln \psi_{r s}+\Psi_{r s}\left(\vartheta_{r} \vartheta_{s}+\varphi_{r} \varphi_{s}\right)+\Omega_{r s} \vartheta_{r} \varphi_{r} \vartheta_{s} \varphi_{s}\right]\right),
\end{aligned}
$$

where the subscript $P$ refers to the planar loop. As at the tree level, the full planar amplitude is given by a sum over noncyclic permutations of external states. The net effect is to remove the $\theta$ functions and integrate $\rho_{r}$ from $\omega$ to 1 .
The result (4.14) is the one-loop amplitude for the case of a NS-sector string propagating around the loop. We must also consider the case of $\mathbf{R}$-sector propagation. The effect is the replacement of $\Psi$ as given by Eq. (4.10) with the fermionic two-point function appropriate to the 
choice of boundary condition. The sum of these expressions yields the full amplitude.

In a similar way, the nonplanar loops can be computed. The only novelty is the introduction of twisting factors between the vertices. ${ }^{37,38}$ When the two external states belong to different boundaries of the annulus, this amounts to replacing the correlation function $\psi$ in Eq. (4.2) by

$$
\psi_{T}(c, \omega)=2 \pi \exp \left(\frac{i \pi v^{2}}{\tau}\right) \frac{\theta_{2}(v, \mid \tau)}{\theta_{1}^{\prime}(0, \mid \tau)}
$$

and $\Psi, \Omega$ in Eq. (4.13) by $\Psi_{T}, \Omega_{T}$, respectively. There is also a change in the domain of the integration of the $\rho_{r}$ : they are integrated over $(-1,-\omega)$ or $(\omega, 1)$. Furthermore, we must sum over the possible choices for NS- or $\mathbf{R}$-sector strings propagating in the loop.

For the case of the nonorientable open toy superstring, we must also include the Möbius loop. Here, the correlation function $\psi$ of Eq. (4.2) is replaced by

$$
\psi_{N}(c, \omega)=-2 \pi i \exp \left(\frac{i \pi v^{2}}{\tau}\right) \frac{\theta_{1}\left(v, \mid \tau+\frac{1}{2}\right)}{\theta_{1}^{\prime}\left(0, \mid \tau+\frac{1}{2}\right)}
$$

and $\Psi, \Omega$ in Eq. (4.13) are replaced by $\Psi_{N}, \Omega_{N}$, respectively. For the Möbius loop, the domain of integration of the $\rho_{R}$ becomes $(-1,-\omega) \cup(\omega, 1)$ and we must sum over the possible choices of NS- or R-sector strings propagating in the loop.

\section{B. Closed superstring}

The computation of the one-loop amplitude for the torus diagram for the closed toy superstring is now straightforward. We begin by considering the amplitude in which a NS-sector string propagates around the loop. The function $\psi$ is replaced by ${ }^{39}$

$$
\begin{aligned}
\chi(c, \omega)= & \exp \left[\frac{\ln ^{2}|c|}{2 \ln |\omega|}\right] \\
& \times\left|\frac{1-c}{\sqrt{c}} \prod_{n=1}^{\infty} \frac{\left(1-\omega^{n} c\right)\left(1-\omega^{n} / c\right)}{\left(1-\omega^{n}\right)^{2}}\right| \\
= & \left.2 \pi \exp \mid-\pi \frac{\operatorname{Im}^{2} v}{\operatorname{Im} \tau}\right)\left|\frac{\theta_{1}(v \mid \tau)}{\theta_{1}^{\prime}(0 \mid \tau)}\right|,
\end{aligned}
$$

and $\Omega, \eta$, and $\Psi$ are replaced by the analogous functions defined in terms of $\chi$ (Ref. 40):

$$
\begin{aligned}
& \Omega^{L L}(c, \omega)=-c \partial_{c} c \partial_{c} \ln \chi(c, \omega) \\
& \Omega^{R R}(c, \omega)=-\bar{c} \partial_{\bar{c}} \bar{c} \partial_{\bar{c}} \ln \chi(c, \omega) \\
& \Omega^{L R}(c, \omega)=\Omega^{R L}(c, \omega)=-c \partial_{c} \bar{c} \partial_{\bar{c}} \ln \chi(c, \omega) \\
& \eta^{L}(c, \omega)=c \partial_{c} \ln \chi(c, \omega) \\
& \eta^{R}(c, \omega)=\bar{c} \partial_{\bar{c}} \ln \chi(c, \omega) \\
& \Psi^{L}(c, \omega)=\eta^{L}(c, \omega)-\eta^{L}(\sqrt{c}, \sqrt{\omega}) \\
& \Psi^{R}(c, \omega)=\eta^{R}(c, \omega)-\eta^{R}(\sqrt{c}, \sqrt{\omega})
\end{aligned}
$$

The four-point amplitude for this spin structure is therefore

$$
\begin{aligned}
A_{T}^{(-+,-+;-+,-+)=\frac{1}{\pi^{4}} \int} \frac{d^{2} \omega}{|\omega|^{2}} \prod_{i=1}^{3} \int_{|\omega|<\left|\rho_{i}\right|<1} \frac{d^{2} \rho_{i}}{\left|\rho_{i}\right|^{2}} \prod_{i=1}^{4} \int d^{2} \vartheta_{i} d^{2} \varphi_{i}\left(\frac{-4 \pi}{\ln |\omega|}\right) \\
\quad \begin{aligned}
\quad \exp \left[\sum _ { r < s } \frac { k _ { r } \cdot k _ { s } } { 2 } \left[\ln \chi_{r s}+\Psi_{r s}^{L}\left(\vartheta_{s} \vartheta_{r}+\varphi_{s} \varphi_{r}\right)+\Psi_{r s}^{R}\left(\bar{\vartheta}_{s} \bar{\vartheta}_{r}+\bar{\varphi}_{s} \bar{\varphi}_{r}\right)\right.\right. \\
\left.\left.+\Omega_{r s}^{L L} \vartheta_{s} \varphi_{s} \vartheta_{r} \varphi_{r}+\Omega_{r s}^{L R} \bar{\vartheta}_{s} \bar{\varphi}_{s} \vartheta_{r} \varphi_{r}+\Omega_{r s}^{R L} \vartheta_{s} \varphi_{s} \bar{\vartheta}_{r} \bar{\varphi}_{r}+\Omega_{r s}^{R R} \bar{\vartheta}_{s} \bar{\varphi}_{s} \bar{\vartheta}_{r} \bar{\varphi}_{r}\right]\right)
\end{aligned}
\end{aligned}
$$

where the superscript denotes the spin structure.

To obtain the complete one-loop amplitude, we must add the contributions from all spin structures. These contributions differ from (4.19) by appropriate replacements for the fermionic two-point functions.

Each summand of the full amplitude has the form (4.19). Under the transformation $v \rightarrow v+1$ and $v \rightarrow v+\tau$, the function $\chi$ and the bosonic functions $\Omega$ are invariant. Furthermore, the fermionic functions change at most by a sign, and they occur only in even powers. Thus, the expression (4.19) is invariant. The region of integration of $\rho$ is therefore restricted so that $0 \leq \operatorname{Im}\left(v_{i}\right) \leq \operatorname{Im}(\tau)$ and $-\frac{1}{2} \leq \operatorname{Re}\left(v_{i}\right) \leq \frac{1}{2}$.

The modular invariance of the full amplitude is verified in the standard way. The invariance of the individual amplitudes of the form (4.19) under $\tau \rightarrow \tau+1, v \rightarrow v$ is immediate. Consider then the behavior of these amplitudes under the modular transformation $v \rightarrow \widetilde{v}=v / \tau$, 
$\tau \rightarrow \widetilde{\tau}=-1 / \tau$. The function $k_{k_{r}} \mathcal{k}_{s} / 2$ becomes $\chi(\widetilde{\boldsymbol{v}}, \widetilde{\tau})$ $=\chi(\nu, \tau) /|\tau|$. The factor $\prod_{r<s} \chi_{r s}^{k_{r} \cdot k_{s} / 2}$ is modular invariant because $\sum_{r<s} k_{r} \cdot k_{s}=0$. Other sources of powers of $|\tau|$ are as follows. The functions $\Omega^{L L}, \Omega^{L R}, \Omega^{R L}, \Omega^{R R}$ change according to $c \partial_{c} \ln \chi(c, \omega)=(1 / 2 \pi i) \partial_{\gamma} \ln \dot{\chi}$ $\rightarrow \tau(1 / 2 \pi i) \partial_{\tilde{\gamma}} \ln \chi$ and its complex conjugate. For a given spin structure $(\widetilde{S}, S)$, the functions $\Psi^{L,(\tilde{S})}$ and $\Psi^{R,(S)}$ transform into $(1 / \tau) \Psi^{L,\left(\tilde{S}^{\prime}\right)}$ and $(1 / \widetilde{\tau}) \Psi^{R,\left(S^{\prime}\right)}$. Since the functions $\widetilde{S}^{\prime}(\widetilde{S})$ and $S^{\prime}(S)$ are one-to-one maps, the spin structures become relabeled in the sum for the full amplitude. Moreover, there is a factor $\tau$ for any pair of Grassmann-valued variables. The integration over the latter leaves a factor $|\tau|^{2 \times 1 / 2 \times 4 \times 2}$. The measures $d^{2} \tau$ and $d^{2} v_{i}$ 's give a factor $|\tau|^{-4-2 \cdot 3}$. Finally, $\left(-8 \pi^{2} /\right.$ $\ln |\omega|)=4 \pi / \operatorname{Im} \tau$ becomes $(4 \pi / \operatorname{Im} \tau)|\tau|^{2}$. Combining the above, we see that no extra power of $|\tau|$ is introduced in the integrations of type (4.19) by the above modular transformation. We conclude that the full amplitude is modular invariant.

To avoid overcounting, the integration in Eq. (4.19) must be restricted so that $\tau$ spans a fundamental region of the modular group, for instance, $|\tau| \geq 1,-\frac{1}{2} \leq \operatorname{Re}(\tau) \leq \frac{1}{2}$.

Modular invariance in the full closed $N=2$ superstring has been considered in Ref. 9, where it was found that the vacuum amplitudes are modular invariant for each spin structure separately. This conclusion also holds for our closed toy superstrings; however, modular invariance of one-loop amplitudes with external states requires a sum over spin structures.

\section{Heterotic strings}

The computation of the one-loop scattering amplitude for the heterotic string follows the standard pattern. ${ }^{11}$ We start by considering the scattering of four charged states in the bosonic description. At one-loop level the new feature is the trace over the internal symmetry states. The zero-mode piece contributes to the sum

$$
Z=\sum_{\lambda \in \Lambda} \exp \left[\frac{1}{2} \ln \omega\left[\lambda+\sum_{r=1}^{4} \frac{\ln \rho_{r}}{\ln \omega} h_{r}\right]^{2}\right]
$$

over the lattice points. Similarly, there is a lattice sum for the $Y_{R}$ coordinates:

$$
Z_{0}=\sum_{\lambda_{0} \in \Lambda_{0}} \exp \left[\frac{1}{2} \ln \bar{\omega}\left[\lambda_{0}+\sum_{r=1}^{4} \frac{k_{r}}{\ln \bar{\omega}} \vartheta_{r} \varphi_{r}\right)^{2}\right]
$$

The amplitude for four charged external states is

$$
\begin{aligned}
& A_{(c)}^{\mathrm{het}}=\frac{1}{(4 \pi)^{4}} \epsilon \int \frac{d^{2} \omega}{|\omega|^{2}} \prod_{i=1}^{3} \int \frac{d^{2} \rho_{i}}{\left|\rho_{i}\right|^{2}} \frac{-4 \pi}{\ln |\omega|} \prod_{r<s} \chi_{r s}^{k_{r} \cdot k_{s} / 2} \\
& \times \prod_{i=1}^{4}\left[\int d \vartheta_{i} d \varphi_{i}\right) Z_{0} \exp \left(\sum_{r<s} k_{r} \cdot k_{s}\left[\Psi_{r s}^{R}\left(\vartheta_{r} \vartheta_{s}+\varphi_{r} \varphi_{s}\right)-\Omega_{r s} \vartheta_{r} \vartheta_{s} \varphi_{r} \varphi_{s}\right]\right) \\
& \times \frac{1}{\omega} \frac{1}{f(\omega)^{24}} \prod_{r<s} \psi_{r s}^{h_{r} \cdot h_{s}},
\end{aligned}
$$

where $\epsilon$ is the cocycle factor associated with the internal momenta $h_{r}$ of the states and where $\psi$ is given by Eq. (4.2), $\Omega$ by (4.7), $\chi$ by (4.17), and $\Psi^{R}$ by (4.18).

As for the closed string, the complete amplitude is obtained summing over the possible spin structures for the rightmoving fermions. Each amplitude of the form (4.22) is invariant under $v \rightarrow v+1$ and $v \rightarrow v+\tau$. Furthermore, the full amplitude is modular invariant, so that the integration in Eq. (4.22) is restricted to a fundamental region of the modular goup, as for the closed string.

The proof follows the general pattern described for the closed-string amplitude. Note that the quantity $Z_{0}$ provides a contribution canceling the zero-mode factor from the transformation of $\Omega$, while $Z$ provides one compensating for the change in $\omega^{-1} f(\omega)^{-24}$ and $\psi$. In addition, to have the correct transformation properties under the modular group, the lattices $\Lambda$ and $\Lambda_{0}$ must be self-dual. Since $p^{Y}=0$ at the massless level, the vectors associated with the internal states must belong to an even self-dual sublattice of $\Lambda$. Thus, possible symmetries of the massless states in the toy heterotic superstrings are those with algebra roots lying on a Niemeier lattice. ${ }^{41}$

Next, we consider the one-loop amplitude in the fermionic description. The external states are described by polarization matrices $T^{i j}$, Eq. (2.38). This results in the factor

$$
\frac{1}{16} \sum_{\{n\}\{p\}} T^{i_{1} j_{1}} \cdots T^{i_{4} j_{4}} \operatorname{tr}\left(\psi_{n_{1}}^{i_{1}} \psi_{p_{1}}^{j_{1}} \cdots \psi_{n_{4}}^{i_{4}} \psi_{p_{4}}^{j_{4}} \omega^{\tilde{L}_{F, 0}}\right) \rho_{1}^{-n_{1}-p_{1}} \cdots \rho_{4}^{-n_{4}-p_{4}} .
$$

Since $i_{r} \neq j_{r}$, the $n$ and $p$ indices are paired to give either a product of two traces of two T's or a trace of four T's. The former provides factors of the type

$$
\frac{1}{16} \operatorname{tr}\left(T_{1} T_{2}\right) \operatorname{tr}\left(T_{3} T_{4}\right) \prod_{(r, s)}\left(\sum_{h=1 / 2}^{\infty} \frac{c_{s r}^{h}-\omega^{h} / c_{s r}^{h}}{1-\omega^{h}}\right]^{2},
$$

where the product is over the pairs $(r, s)=(1,2)$ and $(3,4)$. Similarly, the latter provides factors of the form 


$$
-\frac{2}{16} \operatorname{tr}\left(T_{1} T_{2} T_{3} T_{4}\right) \prod_{(r, s)} \sum_{h=1 / 2}^{\infty} \frac{c_{s r}^{h}-\omega^{h} / c_{s r}^{h}}{1-\omega^{h}}
$$

where the product is over all pairs $(r, s)=(1,2),(2,3),(3,4)$, and $(1,4)$.

The resulting amplitude for one spin structure is

$$
\begin{aligned}
& A^{\text {het }}=\frac{1}{(4 \pi)^{4}} \int \frac{d^{2} \omega}{|\omega|^{2}} \prod_{r} \int \frac{d^{2} \rho_{r}}{\left|\rho_{r}\right|^{2}} \frac{-4 \pi}{\ln |\omega|} \prod_{r<s} \chi_{r s}^{k_{r} \cdot k_{s} / 2} \prod_{r}\left[\int d \vartheta_{r} d \varphi_{r}\right] Z_{0} \exp \left[\sum_{r<s} k_{r} \cdot k_{s}\left[\Psi_{r s}^{R}\left(\vartheta_{s} \vartheta_{r}+\varphi_{s} \varphi_{r}\right)-\Omega_{r s} \vartheta_{s} \varphi_{s} \vartheta_{r} \varphi_{r}\right]\right] \\
& \times \frac{1}{16} \frac{P}{\omega}\left[\operatorname{tr}\left(T_{1} T_{2}\right) \operatorname{tr}\left(T_{3} T_{4}\right) \Psi_{12}^{2} \Psi_{34}^{2}-2 \operatorname{tr}\left(T_{1} T_{2} T_{3} T_{4}\right) \Psi_{12} \Psi_{23} \Psi_{34} \Psi_{14}+\text { cyclic }\right],
\end{aligned}
$$

where

$$
P=\prod_{r=1 / 2}^{\infty}\left(1-\omega^{r}\right)^{48}
$$

The complete amplitude is obtained by summing over spin structures. The whole sum is modular invariant, and the integration in each summand of the form (4.26) is restricted to a fundamental region of the modular group.

This amplitude has the same structure as the tree-level amplitude (3.20). There are terms describing the exchange of an intermediate state and others associated with disconnected diagrams in the 26-dimensional sector.

\section{CONCLUSION}

In this paper, we have formulated and explored the basic properties of several toy superstring models. These are models with critical dimension two that have only two real bosonic spacetime coordinates. Apart from the intrinsic interest of toy superstrings, simple models of this type may ultimately yield insights into the physics of ten-dimensional superstring theories.

The toy superstrings are constructed by a projection defined via toroidal compactification from twodimensional models with two $X$ and two $Y$ coordinates. In our closed-string models, these coordinates are further restricted to be periodic. The models discussed include open superstrings, closed superstrings, and $(2,0)$ heterotic strings.

The spectrum and dimensionality for each toy model is analyzed via conformal methods. Spin fields are introduced and vertex operators are obtained. Tree-level and one-loop scattering amplitudes are derived. The closed and heterotic toy superstring amplitudes are modular invariant to one-loop order.

\section{ACKNOWLEDGMENTS}

We thank S. Samuel for useful discussions. This research was supported in part by the United States Department of Energy under Contract Nos. DE-AC0284ER40125 and DE-AC02-86ER40253.
${ }^{1}$ See, for example, J. Scherk, Rev. Mod. Phys. 47, 123 (1975); J. H. Schwarz, Phys. Rep. 89, 223 (1982); Superstrings, edited by J. H. Schwarz (World Scientific, Singapore, 1985), Vols. I and II; Unified String Theories, edited by M. B. Green and D. J. Gross (World Scientific, Singapore, 1986).

${ }^{2}$ M. B. Green, J. H. Schwarz, and E. Witten, Superstring Theory (Cambridge University Press, Cambridge, England, 1987), Vols. I and II.

${ }^{3}$ M. Ademollo et al., Nucl. Phys. B111, 77 (1976).

${ }^{4}$ M. Ademollo et al., Phys. Lett. 62B, 105 (1976).

${ }^{5}$ L. Brink and J. H. Schwarz, Nucl. Phys. B121, 289 (1977).

${ }^{6}$ A. D'Adda and F. Lizzi, Phys. Lett. B 191, 85 (1987).

${ }^{7}$ M. B. Green, Phys. Lett. B 193, 439 (1987); Nucl. Phys. B293, 593 (1987).

${ }^{8}$ S. D. Mathur and S. Mukhi, Phys. Rev. D 36, 465 (1987).

${ }^{9}$ S. D. Mathur and S. Mukhi, Nucl. Phys. B302, 130 (1988).

${ }^{10}$ E. S. Fradkin and A. A. Tseitlin, Phys. Lett. 162B, 295 (1985);

A. A. Tseitlin, Pis'ma Zh. Eksp. Teor. Fiz. 42, 432 (1985)
[JETP Lett. 42, 535 (1985)].

${ }^{11}$ D. J. Gross, J. A. Harvey, E. Martinec, and R. Rohm, Nucl. Phys. B256, 253 (1985); B267, 75 (1986).

12J. E. Paton and Chan H.-M., Nucl. Phys. B10, 516 (1969); N. Marcus and A. Sagnotti, Phys. Lett. 119B, 97 (1982).

13J.-L. Gervais and B. Sakita, Nucl. Phys. B34, 477 (1971).

${ }^{14}$ L. Brink, D. I. Olive, and J. Scherk, Nucl. Phys. B61, 173 (1973).

${ }^{15}$ A. A. Belavin, A. M. Polyakov, and A. B. Zamolodchikov, Nucl. Phys. B241, 333 (1984).

${ }^{16}$ V. G. Knizhnik and A. B. Zamolodchikov, Nucl. Phys. B247, 83 (1984).

${ }^{17}$ M. A. Bershadsky, V. G. Knizhnik, and M. G. Teitelman, Phys. Lett. 151B, 31 (1985).

${ }^{18}$ D. Friedan, Z. Qiu, and S. Shenker, Phys. Lett. 151B, 37 (1985).

${ }^{19}$ D. Friedan, S. Shenker, and E. Martinec, Phys. Lett. 160B, 55 (1985). 
${ }^{20}$ V. G. Knizhnik, Phys. Lett. 160B, 403 (1985).

${ }^{21}$ D. Friedan, E. Martinec, and S. Shenker, Nucl. Phys. B271, 93 (1986).

22J. Cohn, D. Friedan, Z. Qiu, and S. Shenker, Nucl. Phys. B278, 577 (1986).

${ }^{23}$ V. A. Kostelecký, O. Lechtenfeld, W. Lerche, S. Samuel, and S. Watamura, Nucl. Phys. B288, 173 (1987).

${ }^{24}$ A. R. Bogojevic and Z. Hlousek, Phys. Lett. B 179, 69 (1986).

${ }^{25}$ A. Bilal, Phys. Lett. B 180, 255 (1986).

${ }^{26}$ P. Goddard and D. I. Olive (unpublished).

${ }^{27}$ C. B. Thorn, Phys. Rev. D 4, 1112 (1971).

${ }^{28}$ J. H. Schwarz, Phys. Lett. 37B, 315 (1971).

${ }^{29}$ E. F. Corrigan and D. I. Olive, Nuovo Cimento 11A, 749 (1972); 18A, 339 (1973).

${ }^{30}$ E. F. Corrigan and P. Goddard, Nucl. Phys. B68, 189 (1974).

${ }^{31}$ M. B. Green and J. H. Schwarz, Nucl. Phys. B181, 502 (1981); B198, 441 (1982); B198, 252 (1982); Phys. Lett. 109B, 444 (1982).

${ }^{32}$ M. B. Green, J. H. Schwarz, and L. Brink, Nucl. Phys. B198,
474 (1982).

${ }^{33} \mathrm{~A}$ more general form for the vertex, such as $W^{\prime}=: \exp [i k \cdot(\cos \phi X+\sin \phi Y)]:$, could be considered. However, this is equivalent under a global $\mathrm{O}(2)$ rotation to $W$ and yields the same amplitudes.

${ }^{34}$ A. Neveu, J. H. Schwarz, and C. B. Thorn, Phys. Lett. 35B, 529 (1971).

${ }^{35}$ Here and in the following, we adopt the notation of Ref. 22 for $\psi, \Psi, \Omega, \eta$.

${ }^{36}$ Higher Transcendental Functions (Bateman Manuscript Project), edited by A. Erdelyi et al. (McGraw-Hill, New York, 1953), Vol. II.

${ }^{37}$ L. Caneschi, A. Schwimmer, and G. Veneziano, Phys. Lett. 30B, 351 (1969).

${ }^{38}$ D. Amati, M. Le Bellac, and D. I. Olive, Nuovo Cimento 66A, 831 (1970).

${ }^{39}$ J. A. Shapiro, Phys. Rev. D 5, 1945 (1972).

${ }^{40}$ These are denoted $\hat{\Omega}$ and $\hat{\eta}$ in Ref. 22.

${ }^{41}$ H. Niemeier, J. Number Theory 5, 142 (1973). 